\title{
BIOTECNOLOGÍA Y SISTEMAS DE SALUD EN LOS PAÍSES EN VÍA DE DESARROLLO*
}

\author{
Diana Rocío Bernal Camargo, Ph.D.** Nancy Milena Bernal Camargo, MD ***
}

\begin{abstract}
Resumen
Se analizan las relaciones y los retos que surgen entre la investigación académica y la industria biotecnológica en la promoción del acceso a esta tecnología aplicada a la salud en países en vía de desarrollo, partiendo de una revisión teórica sobre las relaciones y los nuevos conflictos de interés que aparecen entre biotecnología, industria y academia. Se estudian en forma crítica informes y reportes sobre la situación planteada para evidenciar los retos desde la perspectiva de la bioética. Algunos de estos países están iniciando procesos de investigación en biotecnología aplicada a la salud, pero es lamentable que aun no alcancen niveles de cobertura deseable ni se generen a través de procesos de innovación desarrollados al interior, como producto de alianzas estratégicas entre academia-industria y estado. Se debe propiciar un avance hacia la inversión en investigación e innovación en esta tecnología para superar el estado de la medicina curativa y así llegar a una de tipo predictiva -coetánea a la preventiva-como proceso efectivo del disfrute de los más altos estándares del derecho a la salud y se avance en un sistema de generación de políticas públicas a partir de resultados concretos de investigaciones multidisciplinarias en el área de la salud.
\end{abstract}

Palabras clave: biotecnología, bioética, salud, países en desarrollo, cooperación.

\section{BIOTECHNOLOGY AND HEALTH SYSTEMS IN DEVELOPING COUNTRIES}

\begin{abstract}
We analyzed relationships and challenges arising between academic research and the biotechnology industry on promoting access to health biotechnology in developing countries. First, a theoretical review on the relationships and new conflicts of interest emerging between biotechnology, industry and academic research was performed. Statements and reports on this situation were critically studied in order to evidence the challenges from a bioethics perspective. Some of these countries are beginning to conduct research processes on health biotechnology, but it is deplorable they do not yet achieve targeted coverage levels through innovative processes developed from within as a result of strategic partnerships between academic research, industry and state. Investment should be directed towards research and innovation in this type of technology in order to enable overcoming a curative type of medicine and introducing a predictive type of healthcare system -coetaneous to preventive medicine- as an effective process which translates in enjoying the highest standards of the right to health and progressing to a system which legislates public policies derived from concrete multidisciplinary research on health issues results.
\end{abstract}

Key words: biotechnology, bioethics, health, developing countries, cooperation.

Fecha recibido: mayo 14 de 2012 - Fecha aceptado: octubre 26 de 2012

* Articulo que hace parte del proyecto de investigación "Perspectiva bioética de la salud y su relación con otros derechos humanos en países en vía de desarrollo" y se encuentra adscrito a la línea de investigación "Políticas Públicas Sociales y Seguimiento Normativo" del Grupo de Investigación en Derechos Humanos, Facultad de Jurisprudencia, Universidad del Rosario. Bogotá DC, Colombia.
* Abogada, Doctora en Derecho (linea en Bioética y Derechos Humanos). Profesora-investigadora de carrera, Grupo de Investigación en Derechos Humanos, Facultad de Jurisprudencia, Universidad del Rosario, Bogotá DC, Colombia.

*** Médica epidemióloga. Residente III de pediatria, Fundación Universitaria Ciencias de la Salud. Bogotá DC, Colombia. 


\section{Introducción}

Los actuales avances de la ciencia y la tecnología al servicio de la sociedad plantean un importante cuestionamiento respecto a ¿qué tipo de relaciones se generan entre la investigación académica y la industria biotecnológica, que permitan promover el acceso de esta última a los sistemas de salud de los países en vía de desarrollo? Se plantea la necesidad de promover una relación de cooperación en términos de equidad entre los procesos de investigación que desarrollan el sector académico y la industria biotecnológica para el acceso a los estándares más altos de salud, con el fin de contribuir a la solución de conflictos que aparecen ante la tecnología aplicada para la prevención o el tratamiento de enfermedades, y en general para el mejoramiento de la calidad de vida de la población. En este sentido se hace necesario indagar por la construcción de políticas públicas hacia la generación y aplicación de la biotecnología en los sistemas de salud de países en desarrollo, en especial cuando se debe repensar el significado de la cooperación internacional o las alianzas mundiales en este campo.

\section{Metodología}

Se realizó una revisión y exploración de informes y reportes mundiales sobre el estado de la biotecnología y su aplicación a la salud para contrastarlos con los adelantos y/o avances que sobre este punto se encuentran en los países desarrollados. Se encuentran dos interrogantes como son: ¿cómo incorporar la biotecnología en países en vía de desarrollo a un bajo costo para los estados? y ¿cómo permitir el acceso de la mayor parte de la población a esa biotecnología y no solo a las personas con mayores recursos económicos? Para ello se analizan en primer lugar las relaciones y conflictos que generan los avances en biotecnología; después se abordan elementos de la incorporación de la biotecnología en los sistemas de salud de los países en vía de desarrollo, para así por último analizar las perspectivas y retos de la relación academia-industria en investigación, para contar con la biotecnología en los sistemas de salud en países en vía de desarrollo.

\section{Relaciones y conflictos que generan los avances en biotecnología}

Ha sido tradicional que los conflictos de interés en el área de la salud se han circunscrito a la toma de decisiones en la relación médico-paciente, la cual se vio transformada con la superación de la medicina paternalista y el fortalecimiento de la autonomía de los pacientes.

Cuando se habla de conflicto de interés se hace referencia a circunstancias en las que los intereses personales aparecen en oposición a los deberes profesionales. ${ }^{1}$ En la relación paternalista el médico toma todas las decisiones en nombre del enfermo sin tener en cuenta su consentimiento, so pretexto de actuar en favor de aquel. En esta relación el médico puede manipularlo y no respetarle su autonomía, por cuanto entiende que posee autoridad científica y moral, mientras que el paciente no puede decidir por su ignorancia en la medicina, sin tener en cuenta que el profesional tiene la obligación de explicar los conocimientos necesarios al paciente para que participe en la toma de decisiones. ${ }^{2}$

Con la incursión del principio de la autonomía y del consentimiento informado en la práctica médica, en especial a través del desarrollo del sistema legal norteamericano debido al auge de las demandas de pacientes contra médicos por malpractice a partir de la década de los $70^{3}$, se generó una transformación de los conflictos de intereses y de la participación de los pacientes. Este cambio se vio fortalecido con la aparición de las primeras Cartas de Derechos de los Pacientes, originadas en los ciudadanos norteamericanos que buscaban ser tenidos en cuenta como sujetos activos y participativos, y fue así como esta necesidad se extendió a diferentes latitudes del mundo.

Debido a ese llamado por el respeto a los derechos de los pacientes, los conflictos de interés surgen por la "divergencia entre beneficios personales o institucionales comparados con la responsabilidad hacia los pacientes y la sociedad en el contexto de la investigación, la inversión financiera y el liderazgo en el manejo de los servicios médicos". ${ }^{4}$ 
Sin embargo la sociedad, la práctica médica y en consecuencia sus conflictos de interés, en la actualidad enfrentan diversos desafíos originados en los últimos descubrimientos científicos, las nuevas tecnologías aplicadas a la vida y las necesidades de la población que surgen en relación con nuevos estándares de calidad de vida.

Asistimos a una nueva era de desarrollo científico y tecnológico, que podría llamarse como la "era biotecnológica". En términos generales, se puede entender como el uso de organismos vivos o sustancias biológicas para el desarrollo de procesos industriales o la fabricación de productos de diagnóstico o terapéuticos, como el caso de la ingeniería genética. La biotecnología no es novedosa ya que se practica desde la antigüedad; sin embargo, ahora tiene aplicaciones muy variadas no solo en agricultura y producción de alimentos, sino también en relación con la protección y conservación de medio ambiente, y en el campo de la salud en el descubrimiento y desarrollo de nuevos medicamentos para la prevención o el tratamiento de enfermedades (biofarmacia), así como para el diagnóstico de éstas, llegando a niveles como la manipulación del ADN, que es la forma más moderna de la utilización de organismos vivos a través de la ingeniería genética (clonación, terapia génica, etc). ${ }^{5}$

Las aplicaciones actuales de la biotecnología junto con el desarrollo de la genómica, podría entenderse como un tipo especial de revolución científica, en el sentido que han cambiado ciertos paradigmas y han dado un giro insospechado al curso de las investigaciones científicas, sobre todo en los países desarrollados en los que el uso de la biotecnología se ve como la alternativa ideal para la solución de problemas, como la escasez de alimentos o la cura de enfermedades que hasta ahora parecía imposible.

Sin embargo, el desarrollo de esta biotecnología ha ahondado las distancias entre aquellos y los países en vía de desarrollo y subdesarrollados, en donde la producción y el acceso a esa nueva biotecnología genera conflictos tecnocientíficos, sociales, éticos, culturales, religiosos, morales y en la misma práctica médica. ${ }^{6}$
Sin referirnos a los conflictos bioéticos propios de los nuevos avances en biotecnología ni entrar aquellos relacionados con los países ricos en biodiversidad o con ocasión de la aparición de organismos genéticamente modificados, es de mencionar y resaltar los nuevos conflictos que la biotecnología ha ocasionado en relación con la investigación y el rol de las universidades y la industria, junto a los que se han desarrollado en los sistemas de salud, en especial en los países en vía de desarrollo, en donde hay algunas posibilidades de acceso a la tecnología aplicada a la vida, pero con limitaciones económicas muy importantes.

Es decir, que siguiendo a Henry T. Greely, en relación con la biotecnología encontramos tres tipos de relaciones y de conflictos de interés: a) los que se generan entre las empresas involucradas en la industria biotecnológica, b) los que aparecen entre la industria biotecnológica y el gobierno, y c) los que se originan en las relaciones entre la industria biotecnológica y las universidades ${ }^{7}$, los cuales apuntarían a cuestionamientos en torno a ¿quién y cómo produce la biotecnología? ¿quién y cómo la distribuye? y ¿quién y cómo se beneficia? con la particularidad ya no de los países industrializados, sino de aquellos que están en condiciones menos favorecidas. En este orden de ideas, se verá el contexto de cada uno de estos conflictos.

\section{a) Empresas - industria biotecnológica}

En este punto se toma el caso de las compañías farmacéuticas y de aquellas empresas que desarrollan biotecnología no farmacéutica para el diagnóstico y tratamiento de enfermedades humanas.

La producción de este tipo de tecnología aplicada a la salud humana requiere alianzas con diferentes compañías y sectores, con el fin de incrementar la inversión de capital para encontrar las mejores estrategias para la distribución y venta de los productos obtenidos a partir de este tipo de desarrollo biotecnológico. Se puede decir que en esta categoría se está en presencia de conflictos de interés en especial de tipo económico, en la medida que se encuentra en juego la recuperación o el provecho de las inversiones realizadas por las compañías y empresas que trabajan o invierten en biotecnología. ${ }^{8}$ 
Una de las formas para recuperarestas inversiones es a través de los sistemas de patentes que han ocasionado una serie de controversias, sobre todo en relación con los derechos de propiedad intelectual sobre el cuerpo humano o cualquiera de sus componentes, en donde las compañías patrocinadoras buscan patentar los procesos o los resultados para recuperar esa inversión durante los procesos de investigación. Es decir que el conflicto que se presenta es respecto de si es ético y legal permitir las patentes humanas obtenidas para el diagnóstico y el tratamiento de enfermedades. En términos generales, encontramos dos posiciones: una que indica que no deben permitirse las patentes humanas en tanto que cada individuo tiene intereses continuos de propiedad sobre sus propias células y componentes biológicos, y otra que señala como se ha evolucionado en las decisiones sobre derechos de propiedad intelectual en el sentido en que son muy amplios para quienes desarrollan o trabajan en innovación con células humanas, componentes de estas y en general información celular. ${ }^{9}$ En los casos en los que se ha permitido este tipo de patentes, el argumento ha sido que las células o sus componentes se extraen del cuerpo humano y la persona no tiene derechos de propiedad sobre los mismos, como en el caso de Moore vs Regents of the University of California, sometido a estudio por la Suprema Corte de California y en el que se decidió bajo esta línea argumentativa. ${ }^{10}$

En este orden de ideas, en algunos países desarrollados la ley y la jurisprudencia permiten las patentes sobre material vivo, sea o no humano, controversia que hasta ahora se evidencia en países en vía de desarrollo, en los que está despegando la investigación con fin de obtener patentes sobre material biológico de aplicación a la mejora de la salud, de forma que aquí el conflicto gira en cómo obtener la financiación y con qué tecnología se puede investigar para lograrlo. ${ }^{11,12}$

\section{b) Gobierno - industria biotecnológica}

En los países en vía de desarrollo de América Latina, los gobiernos están haciendo grandes esfuerzos por incluir dentro de sus planes de política nacional estrategias para la financiación de la investigación con recursos públicos y privados para el desarrollo biotecnológico de sus países.

Dentro de los conflictos de intereses que se pueden generar en las relaciones entre gobiernos e industria biotecnológica, se debate si en el caso de la financiación de la investigación por parte de los gobiernos estos deberían tener algún tipo de interés o derechos de propiedad sobre los resultados de las mismas, y si además le asiste el derecho de controlar la comercialización de estas investigaciones que ha financiado. ${ }^{13}$ Sin embargo, en el caso de países en desarrollo se encuentran algunos conflictos previos a los mencionados y que son tanto de tipo económico como legal. Entre los primeros está si la investigación en biotecnología debe ser financiada con recursos públicos y en tal caso en qué porcentaje. En los segundos se encuentran aquellos conflictos relacionados con las normas jurídicas de los países, es decir sobre la flexibilidad de los ordenamientos jurídicos para permitir la investigación científica sin límites legales.

En el caso de América Latina, Brasil y México se muestran como ejemplo de la región en avance de investigación en biotecnología. Otros países que buscan la transferencia de tecnología se encuentran en el proceso de diseño y ejecución de políticas nacionales para la promoción de actividades biotecnológicas, en especial mediante incentivos tributarios ${ }^{14}$, así como la apertura de los estados hacia la inversión extranjera a través de los tratados de libre comercio bajo condiciones de equidad y beneficio real para los países en desarrollo.

\section{c) Universidades - industria biotecnológica}

En los países en vía de desarrollo la relación entre academia e industria en un comienzo no había generado grandes avances, dado el poco o nulo desarrollo científico y tecnológico. Sin embargo, gracias al surgimiento y fortalecimiento de centros de investigación tanto en las facultades de medicina como en instituciones de salud, públicas y privadas, se han creado nuevos lazos incentivados por los gobiernos, en pro del desarrollo de la investigación. 
Así como los avances que han revolucionado la biotecnología son muy recientes, las relaciones entre academia e industria biotecnológica también lo son, en particular en países en desarrollo, en donde los avances en biotecnología están despegando y la investigación académica se encuentra en un proceso de fortalecimiento gracias a la financiación privada y de organismos internacionales, en la que la participación de los gobiernos no es muy considerable aunque inicia un proceso de despegue interesante.

En el sector privado se ha impulsado el desarrollo de la biotecnología como parte de la implementación de los sistemas de investigación y desarrollo (I+D) universitarios, de forma que la investigación en las ciencias de la vida, que era un campo propio de la investigación académica, se ha visto permeada por el sector de los negocios empresariales, por lo que el debate también se ha centrado en el cuestionamiento de ¿sí esta invasión de las empresas en biotecnología ha limitado el acceso a los descubrimientos y en consecuencia se ha disminuido el avance científico? Lo que a su vez trae consigo la cuestión de la ciencia como un negocio de gran rentabilidad. ${ }^{15}$

En este contexto, las investigaciones en ciencias de la vida adelantadas por instituciones de educación superior financiadas por la industria privada, presentan conflictos especiales acordes con la naturaleza de esta relación, entre los que se refieren a las amenazas: a) al carácter fundamental de las empresas de investigación académica, b) a la integridad de los procesos de investigación en sí mismos, que incluyen la objetividad de la investigación y de la enseñanza, y c) a los sujetos humanos de investigación. ${ }^{16}$

En este sentido la apuesta de las relaciones entre investigación académica e industria biotecnológica gira en torno a un tipo particular de relaciones económicas que buscan sacar el mayor provecho, en especial en relación con los derechos de propiedad intelectual, sistema a través del cual se recuperan en forma notable los recursos económicos invertidos en los diferentes procesos de investigación.

\section{La incorporación de la biotecno- logía en los sistemas de salud de los países en vía de desarrollo}

Se debe comenzar por señalar que acorde con un criterio de crecimiento y desarrollo económico, los países pueden clasificarse como desarrollados, en vía de desarrollo y subdesarrollados, que tomando los datos y método del Banco Mundial, corresponden a economías de ingresos alto, medio y bajo. ${ }^{17}$ Sin embargo este índice de desarrollo no siempre corresponde con el nivel de acceso y disfrute al más al to nivel posible de salud, y en consecuencia de la tecnología desarrollada para la consecución de tal fin.

Con ocasión de los costos que genera la producción de biotecnología, resulta oportuno hacer referencia a cómo debe incorporarse su aplicación a los sistemas de salud, que en países desarrollados en principio no genera controversia, pero en aquellos en vía de desarrollo causa una nueva fase de conflictos de interés en la práctica médica, ética y jurídica.

El caso de los sistemas de salud de los países en vía de desarrollo es de gran interés por cuanto aún se están resolviendo problemas de la falta de cobertura de los planes básicos en salud, a lo que se agrega que los nuevos desarrollos en biotecnología están aún lejos de llegar a estos planes y en consecuencia ello origina nuevos retos que deben afrontarse con el fin de ampliar los servicios en pro de la calidad de vida y así brindar servicios eficientes como objetivo fundamental de los sistemas de salud. ${ }^{18.19}$

Algunos informes estiman que existen cerca de " 4.275 empresas biotecnológicas en el mundo, de ellas el $64 \%$ trabaja en la salud humana, $45 \%$ están en Norteamérica, 38\% en Europa y $17 \%$ en Asia y el Pacifico"20, en un contexto en el que la cooperación constituye un eje de gran importancia para direccionar el desarrollo biotecnológico de las regiones en vía de desarrollo y las subdesarrolladas y contribuir a la reducción de la pobreza, aunque también se debe tomar en cuenta la otra lectura que de esta relación se hace en diferentes escenarios, la cual señala que la estructura de 
estas relaciones entre países desarrollados y en vía de desarrollo y subdesarrollados buscan favorecer las economías desarrolladas en donde "el subdesarrollo y el desarrollo son dos caras de una misma moneda, es decir, como un sistema único que se desarrolla simultáneamente en dos formas opuestas". ${ }^{21}$

Los avances en biotecnología, sin importar que se trate de países desarrollados o subdesarrollados, implican la necesidad de capital humano de gran importancia concentrado tanto en universidades como en centros públicos y privados de investigación, que dependiendo del sector empresarial y la región económica en la que se utilice, se traducirá en resultados patentables. ${ }^{22-24}$ Sin embargo, es claro el desequilibrio que existe entre los desarrollados y subdesarrollados, puesto que las potencias económicas poseen el control sobre los mercados biotecnológicos.

En este panorama se resaltan los avances de países como Brasil, India, México y Cuba, en donde la estrategia empleada ha sido el fortalecimiento de asociaciones público-privadas para la promoción y el desarrollo de productos y tratamientos de enfermedades en países en vía de desarrollo. No obstante, en estas la industria híbrida en biotecnología ha tenido un gran inconveniente puesto que se ha centrado en la investigación de los problemas de salud de los países avanzados dejando de lado los locales. ${ }^{25} \mathrm{Sin}$ embargo, se debe resaltar la importancia que traen las investigaciones biotecnológicas en el campo de la genómica, a través de las cuales se pueden mejorar o agudizar las desigualdades globales en salud, teniendo en cuenta que son precisamente los países en desarrollo los que quedan rezagados en el desarrollo de estas nuevas tecnologías y en el avance de la medicina genómica, por lo cual, incluso dentro de los Objetivos del Milenio se ha propuesto, entre otros al Centro Asociado de Bioética de la Universidad de Toronto, participar en un grupo de trabajo de genómica "para la mejora de la salud global a través de la biotecnología genómica" 26 , el cual se ha iniciado en siete países en desarrollo: Brasil, China, Cuba, Egipto, India, Sudáfrica y Sur Corea, lo cual se considera que pueden servir de ejemplo para otras naciones en desarrollo, como el caso colombiano.
En este sentido, se debe resaltar que en el marco de los Objetivos de Desarrollo del Milenio en relación con la salud humana, se han tomado como temas de interés prioritario lo relacionado con los avances tecnológicos aplicados sobre todo a vacunas, pruebas de diagnóstico, desarrollo de fármacos y sus sistemas de administración, genómica humana y salubridad ambiental, bajo el entendido de que los progresos en medicina dependen de los adelantos y las aplicaciones de la biotecnología al sector salud, más aún cuando esta se desarrolla para resolver problemas de salud y mejorar la calidad de vida de la población y constituye un componente necesario para la prestación de los servicios en relación con la prevención, el diagnóstico y el tratamiento de enfermedades y en el alivio de las discapacidades y las deficiencias funcionales.

En relación con los medicamentos, dentro de los objetivos del milenio se encuentra en el "Objetivo No. 8 Establecer una alianza mundial para el desarrollo" la meta de "Proporcionar acceso a los medicamentos esenciales a bajo costo en los países en desarrollo en cooperación con las empresas farmacéuticas", sin embargo, del último informe sobre la salud en el mundo de la OMS, señala que en efecto los países afrontan la demanda por mejor y mayor calidad de los servicios en contraste con una alta amenaza de enfermedades y una lista cada vez más voluminosa de tecnologías y medicamentos que suelen ser caros, para mantener o mejorar la salud. Estos costes crecen a mayor ritmo que los ingresos nacionales, es decir que "los nиеvos medicamentos y las nuevas tecnologías terapéuticas surgen mucho más rápido que los nuevos recursos financieros". ${ }^{27}$

El informe señala que en relación con las tecnologías médicas, es imprescindible que se elijan y utilicen en forma adecuada conforme a la evidencia clínica y la práctica médica como quiera que, acorde con el mismo informe: "La tecnología médica moderna es un factor importante en el aumento de los costes de la Organización para la Cooperación y el Desarrollo Económicos (OCDE), y su adquisición en muchos países no siempre está basada en la necesidad. Entre los países de la OCDE, el mayor número de unidades de resonancia magnética ( $R M N)$ y tomografía com- 
putarizada (TAC) per capita se encuentra en Japón, mientras que los Estados Unidos de América es el líder mundial en remisiones al diagnóstico por imagen: 91,2 RMN por cada 1.000 habitantes (en comparación con la media de la OCDE de 41,3 por 1.000) y 227,8 TAC por 1.000 (en comparación con la media de la $O C D E$ de 110). Se cree que una proporción importante de estas pruebas son innecesarias desde el punto de vista médico. La compra y el uso innecesarios de los equipos también se pueden producir en los países de ingresos bajos pero, en general, los centros con pocos recursos tienen otros retos tecnológicos. Se estima que al menos el $50 \%$ de los equipos médicos en los países en desarrollo se utilizan parcialmente o están totalmente inutilizables".

Y más adelante agrega: "Irónicamente, una de las mayores causas de ineficiencia asociada a las tecnologías médicas en los países de ingresos bajos son las donaciones. En algunos países, casi el $80 \%$ de los equipos de atención sanitaria proceden de donantes internacionales o de gobiernos extranjeros y gran parte de ellos permanecen sin funcionar por varias razones. Un ejemplo de ello es el reciente estudio llevado a cabo en Cisjordania y en la Franja de Gaza. ${ }^{27}$ Después de que finalizaran las hostilidades en enero de 2009, se envió un gran cargamento con estos equipos a la Franja de Gaza. Si bien algunos de los equipos donados eran útiles, un porcentaje importante de ellos no pudieron integrarse en el sistema sanitario y se quedaron en los almacenes. Este tipo de problemas se podría evitar si los socios de los países desarrollados consultaran a los países receptores, para aclarar sus necesidades y capacidades y que, de este modo, los equipos donados tengan una utilidad. Los gobiernos receptores también deben establecer sistemas razonables de gestión que organicen el almacenamiento de los productos sanitarios por el tipo, el modelo y el fabricante, y comprueben la integridad, la compatibilidad y la calidad de todo artículo donado".

Uno de los grandes conflictos que se generan con ocasión de la aplicación de la biotecnología en países en desarrollo, además de buscar la financiación y desarrollo de los proyectos de invención y aplicación en biotecnología, es cómo incluir las pruebas diagnósticas, los medicamentos y los tratamientos biotecnológicos en las políticas de cobertura universal, teniendo en cuenta que hay pocos servicios disponibles para todos y que estas pruebas, tratamientos y medicamentos no son de acceso fácil o estable para la población en general y los precios no son razonables.

Esta situación se ve alentada por el hecho de que, si bien dentro de los Objetivos del Milenio se encuentran las alianzas mundiales para el progreso, que permitan el avance de la investigación en biotecnología en los países en desarrollo y no solo la transferencia de productos obtenidos con el fin de que estos países los adquieran y suministren a altos costos en sus sistemas de salud.

Al respecto, es importante referenciar el estudio liderado por la Universidad de Toronto con el fin de establecer el tipo de alianzas y los alcances de la cooperación en el sector de la biotecnología aplicada al sector salud, en especial para determinar si esta cooperación se restringía al comercio o si por el contrario también incluía iniciativas para la investigación, la innovación y desarrollo en biotecnología. Para tal fin se tomaron seis países en desarrollo con sectores biotecnológicos relativamente fuertes: Brasil, China, Cuba, Egipto, India y Sudáfrica, con una participación del $67 \%$ de las empresas dedicadas a biotecnología o a la prestación de servicios producto de desarrollo biotecnológico. En este estudio los investigadores encontraron que un $27 \%$ de estas empresas trabajaba con otros países en biotecnología de la salud, en especial en cooperación sur-norte, pero son predominantes para las últimas etapas de la comercialización (72\%), siendo muy baja la cooperación para la investigación y el desarrollo(13\%). ${ }^{28}$

Esta situación de poca cooperación para investigación y desarrollo se ve alentada por deficiencias en la infraestructura y la falta de apoyo para el desarrollo de productos biotecnológicos de bajo costo. Piénsense en casos como el colombiano en el que el Plan Obligatorio de Salud como norma no se halla respaldado por una política de incorporación de biotecnología para la prestación de servicios en los diferentes regímenes, a lo que se debe agregar que hasta ahora 
en muchos países en desarrollo se está iniciando un proceso de atención con miras a superar la medicina curativa, para avanzar hacia la medicina preventiva, lo que significa que estamos rezagados en relación con la incorporación de biotecnología para así llegar a la medicina predictiva, de forma que aún estamos lejos de incluir en los planes de salud básicos pruebas de mapeo genético, por ejemplo, para así evitar la aparición de la enfermedad y el consecuente gasto en tratamientos curativos.

\section{Perspectivas y retos de la relación academia-industria en investigación para la incorporación de la biotecnología en los sistemas de salud en países en vía de desarrollo}

Las relaciones entre las instituciones académicas y la industria incluyen investigación, sea clínica o no. ${ }^{29}$ Como se ha mencionado, en los países en desarrollo estas relaciones no han generado avances significativos a causa del atraso en el desarrollo científico y tecnológico. Sin embargo, gracias a la creación y fortalecimiento de centros de investigación en diferentes instituciones como las facultades de medicina y las instituciones de salud, públicas y privadas, han aparecido nuevos lazos para la promoción de la investigación por parte de los gobiernos.

A partir del estado actual de la relación entre industria y academia, se plantean nuevos retos para la misma, con el fin de contribuir a la incorporación de la biotecnología a los sistemas de salud, en especial hacia su aplicación en los planes básicos. Esto implica la necesidad de una triangulación en proyectos de investigación entre academia, industria y estado.

Estas relaciones no pueden ser ajenas a los procesos de globalización y al creciente desarrollo de biotecnología liderada por un número no muy amplio de multinacionales. De forma que se requiere la consolidación del trabajo interdisciplinario, que a través de alianzas estratégicas se aborden asuntos de interés público relacionados con el acceso y la calidad de la salud de la población, lo cual suele ligarse a la aplicación de la biotecnología en materia de salud en diferentes campos, por ejemplo alimentaria. En este sentido la globalización también implica un escenario conflictivo en materia de investigación e innovación en el que, si bien se reconoce la soberanía de los estados frente a la presencia de las multinacionales biotecnológicas en sus territorios, lo cierto también es que aún no existe un avanzado desarrollo científico y tecnológico interno en los países en desarrollo, que sin refuerzos será muy difícil salir de la situación de dependencia tecnológica. ${ }^{30}$

Desde esta perspectiva es importante señalar que ello a su vez debe implicar el dialogo inter, multi y transdiciplinario con el fin de que se involucre no solo la medicina, sino otras áreas del conocimiento en procura de la solución de estos nuevos conflictos de intereses, por ejemplo, la interacción de áreas como ingeniería, bioética, derecho, economía y las políticas públicas, entre otras, con el fin de generar propuestas y políticas deseables que permitan el avance de la medicina preventiva a la predictiva, acompañado de programas de educación en salud hacia la población, de forma que en casos como el de la medicina predictiva, se genere una difusión responsable en sus campos relacionados (contenidos genéticos) con el fin de orientar en forma directa e indirecta a la población receptora ${ }^{31}$, para lo cual el papel de las academia es de vital importancia, con el fin de llegar no solo a la población cercana a temáticas académicas del área de la salud, sino al público en general, de ahí la importancia de un trabajo interdisciplinario.

Es evidente que en varios países en desarrollo, la cooperación sur-sur en biotecnología de la salud está pasando de la simple retórica política a la realidad. Las empresas recurren a ella para llegar a mercados no tradicionales y mejorar su competitividad en materia de biotecnología y debería figurar en los programas de más empresas de países en desarrollo. Además, es un medio eficaz de suministrar productos de salud de menor costo, lo que debería interesar a donantes, organizaciones internacionales y filantrópicas. Propiciando la colaboración sur-sur, los donantes pueden aprovechar la capacidad de los países en desarrollo de ofrecer productos más accesibles y útiles a escala 
local y ponerlos a disposición de más personas en el mundo en desarrollo.

Lo anterior va en consonancia con los postulados de la Organización Mundial de la Salud, que en la $58^{\mathrm{a}}$ Asamblea Mundial recalca la necesidad de fomentar en los países en desarrollo la investigación de alta calidad y la generación y aplicación de conocimientos como elementos esenciales para alcanzar los objetivos de desarrollo relacionados con la salud, acorde con la Declaración del Milenio de las Naciones Unidas, razón por la cual se instó a los estados para que destinen al menos el $2 \%$ del presupuesto nacional de salud en investigaciones y en el fortalecimiento de la capacidad de investigación, a través de alianzas estratégicas nacionales, regionales y mundiales, incluyendo sectores público y privado, con el fin de acelerar los procesos de investigación en biotecnología para el desarrollo de medicamentos esenciales, vacunas, pruebas de diagnóstico, tratamiento y mecanismos para la realización equitativa de estas intervenciones. ${ }^{32}$

Es de vital importancia que se tome conciencia sobre la relación que existe entre el acceso universal y equitativo en salud y el fomento a la investigación y la innovación en esta misma en los países en desarrollo, con el fin de que las políticas públicas se formulen a partir de resultados de investigación y no de meros datos sin conclusiones evidentes. En este mismo sentido el informe sobre la salud en el mundo 2012 demostrará que la investigación es útil y esencial para mejorar los resultados sanitarios, más aún cuando, a juicio de esta organización, "La toma de decisiones mal fundamentadas y la falta de confianza en la investigación son algunos de los motivos por los que los servicios no llegan a veces a quienes más los necesitan, por los que los indicadores sanitarios están desfasados y por los que muchos países tienen pocas probabilidades de alcanzar los ODM relacionados con la salud". ${ }^{33}$

\section{Conclusión}

Es de resaltar la importancia que tienen la investigación, la innovación y la biotecnología aplicada a la salud como herramienta para la consecución de los estándares más altos de disfrute del derecho a la salud en los países en desarrollo. Es indispensable que los estados refuercen sus políticas de inclusión e incorporación de la biotecnología a los planes de desarrollo en materia de salud, pero para lograr una cobertura real y amplia de los servicios que surgen se deben generar alianzas estratégicas entre investigación académica, industria biotecnológica y gobiernos. Estas contribuirán a la formulación de políticas públicas que sean producto de los resultados de proyectos de investigación en este campo y a su vez permitirán que el costo de los mismos para la población sea el mínimo.

Una política en este sentido implica repensar la inversión del presupuesto para investigación en los países en desarrollo, con el fin de que la cooperación internacional en materia de desarrollo biotecnológico no se limite a fines comerciales, sino que ayude para que obtengan sus propios productos biotecnológicos.

Con el fin de permitir el acceso a la mayor parte de la población a esa biotecnología y no solo a las personas con mayores recursos económicos, es importante avanzar en el tipo de medicina que queremos para los sistemas de salud de los países en desarrollo, pues en efecto son mayores los costos de aplicación de la biotecnología en el campo de la medicina curativa que en el de la preventiva o la predictiva. Esta labor de construcción de nuevas y mejores políticas públicas en salud puede fortalecerse a través de la creación y consolidación de Comités o Consejos Nacionales de Bioética que promuevan la labor interdisciplinaria y la inclusión de los resultados de proyectos de investigación para la generación de estas políticas.

\section{Referencias}

1. Tonelli M. Conflict of interest in clinical practice. Chest. 2007; Aug; 132 (2): 664-70.

2. Velez LA. Ética médica: interrogantes acerca de la medicina, la vida y la muerte 3a ed. Medellín: Corporación para Investigaciones Biológicas; 2003.

3. Simón P. El Consentimiento Informado. Madrid: Editorial Triacastela; 2000.

4. Camilleri M, Cortese DA. Managing conflict of interest in clinical practice. MayoClin Proc. 2007May; 82(5):607-614.

5. Navarrete VE. Bioethics and biotechnology.Rev. latinoam. Bioet. 2003; 4: 18-35

6. Bota Arqué A. El impacto de la biotecnología en América Latina: espacios de participación social. Acta Bioethi. 2003; 9(1): 21-38. 
7. Greely HA. Conflicts in biotechnology. J Law Med Ethics. 1995; 23:354-59.

8. Ausín T. Conflict of values in pharmaceutical research: between public health and markets. Arbor. 2008; 184 (730): 333-34.

9. Feldman R. Whose body is it anyway? Human cells and the strange effects of property and intellectual property law.StanfordLaw Rev. 2011; 63(6): 13771402 .

10. Human experimentation consent and information consent : Case Compliments of Versuslaw, Fiduciary Duty of Researchers - the Spleen Case - Moore v. Regents of University of California, 793 P.2d 479 (Cal. 1990) [monografía en Internet]. Redmon, WA :VersusLawInc; 1998 [citado 12 Feb 2013]. Disponible en: http:// biotech.law.lsu.edu/cases/consent/Moore_v_Regents.htm

11. Brown AEL. Access to essential technologies: The role of the interface between intellectual property, competition and human rights. Int.Review Law, ComputTechn. 2010;24(1):51-61

12. Kevles DJ. From eugenics to patents: Genetics, law, and human rights. Ann Hum Genet. 2011 May;75(3):326-33

13. Greely HA. Conflicts in biotechnology. J Law Med Ethics. 1995; 23:356.

14. Jarrett SW. Challenges to the successful introduction of biotechnologies in developing countries. Public Health Ethics; 2008;1(2):104-109.

15. Pisano GP. Can Science Be a Business? Lessons from Biotech.Harv Buss Rev. 2006; Oct: 1-14.

16. Toneguzzo F. Tensions in industry-academic collaborations: perspective of the academic medical center. Asia Pacific Biotech News.2004; 8(10):569-77.

17. World Bank. How we classify countries? Country and Lending groups [monografía en Internet]. Washington DC: World Bank; 2012 [citadol5 Feb 2013]. Disponible en: http://data.worldbank.org/about/country-classifications/countryand-lending-groups

18. Antoniol G. Sistemas de salud en América Latina: retos y funciones. Revista de antiguos alumnos del IEEM. 2004; 12: 84-88.

19. UN.General Assembly.Human rights guidelines for pharmaceutical companies in relation to access to medicines.[monografía en Internet]. Ginebra, Suiza: OACDH; 2008. [citado 9 Jul 2002]. Disponible en:http://www.ohchr.org/Documents/Issues/Health/GuidelinesForPharmaceuticalCompanies.doc.

20. Núñez L, Rojas L, Campo S, Castro JA. Retos y contradicciones de la industria biotecnológica actual. Biotecnol.Apl. 2007; 24: 276-82
21. Olmedo R. Introducción a las teorías sobre el subdesarrollo. En AAV. Feudalismo, capitalismo. Madrid, España: Akal Editor; 1977.

22. González CA, Villa Latorre JM, Bravo JE. El Potencial empresarial de la biotecnología. Rev.Biotecnol. Sector Agrop. Agroind.2007; 5 (2): 112-117.

23. Paul P, Mukhopadhyay K. Growth via intellectual property rights versus gendered inequity in emerging economies: An ethical dilemma for international business. J Bus Ethics. 2010; 91(3):359-78.

24. Rangnekar D.The challenge of intellectual property rights and social justice.Development. 2011; 54(2): 212-14.

25. Thorsteinsdóttir $\mathrm{H}$. The role of the health system in health biotechnology in developing countries.J Technol Analysis Strategic Management. 2007 Sept; 19 (5): 659-75.

26. DaarAbdallah S, Sahni Puja, Singer Peter A. Genomics, biotechnology and globalhealth: the work of the University of Toronto Joint Centre for bioethics. Acta Bioeth. 2004; 10(2):213-25.

27. OMS. Informe sobre la salud en el mundo: la financiación de los sistemas de salud, el camino hacia la cobertura universal [monografía en Internet]. Ginebra, Suiza: OMS; 2011. [citado 9 Jul 2002]. Disponible en:http://whqlibdoc.who.int/ whr/2010/9789243564029 spa.pdf,

28. Thorsteinsdóttir H, Melon C, Ray M, Chakkalackal S, Li M, Cooper J, Singer P. et al. South-South entrepreneurial collaboration in health biotech.Nat Biotechnol. 2010 May; Volume:28(5): Pages: 407-16

29. Blumenthal D. Academic-industrial relationships in the life sciences.N Engl J Med. 2003 Dec 18;349(25):2452-9.

30. Pérez Pérez JA. La bioética y la biotecnología, una mirada hacia el medio ambiente. Ágora. 2006 Ene-Jun; 9: 81-88.

31. Castiel LD, Sanz Valero J. Pruebas genéticas predictivas y percepción de riesgos. Apuntes para la comunicación pública de contenidos sobre biotecnología. Revista Salud Colect. 2006; Mayo-Ago; 2(2): 161-72.

32. OMS. Asamblea Mundial de la Salud.Resolución WHA58.34 Cumbre ministerial sobre investigación en salud (novena sesión plenaria, 25 de mayo de 2005, Comisión A, octavo informe).

33. OMS. Elementos preparatorios informe sobre la salud en el mundo 2012 [monografía en Internet]. Ginebra, Suiza: OMS; 2012. [citado 12Feb 2013]. Disponible en:http://www.who.int/rpc/whr2012-stories/es/index.html 